\title{
Discharge Information and Support for Patients Discharged from the Emergency Department: Results from a Randomized Controlled Trial
}

\author{
Susan N. Hastings, MD, MHSc $c^{1,2,3,4,5}$, Karen M. Stechuchak, MS', \\ Cynthia J. Coffman, $P h D^{1,6}$, Elizabeth P. Mahanna, $M P H^{7}$, Morris Weinberger, $P h D^{1,7}$, \\ Courtney H. Van Houtven, PhD ${ }^{1,5}$, Kenneth E. Schmader, $M D^{1,2,3,4}$, \\ Cristina C. Hendrix, DNS, GNP-BC, FAAN ${ }^{3,9}$, Chad Kessler, MD ${ }^{1,2}$, \\ Jaime M. Hughes, $P h D, M P H, M S W^{1,4,5}$, Katherine Ramos, $P h D^{1,3,4,8}$, \\ G. Darryl Wieland, $\mathrm{PhD}, \mathrm{MPH} \mathrm{H}^{3,4}$, Madeline Weiner, $R N^{3}$, Katina Robinson, $\mathrm{MS}^{7}$, and \\ Eugene Oddone, $\mathrm{MD}, \mathrm{MHSc} \mathrm{C}^{1,2}$
}

\begin{abstract}
${ }^{1}$ Center of Innovation to Accelerate Discovery and Practice Transformation, Durham VA Health Care System, Durham, NC, USA; ${ }^{2}$ Department of Medicine, Duke University School of Medicine, Durham, NC, USA; ${ }^{3}$ Geriatric Research, Education, and Clinical Center, Durham VA Health Care System, Durham, NC, USA; ${ }^{4}$ Center for the Study of Human Aging and Development, Duke University, Durham, NC, USA; ${ }^{5}$ Department of Population Health Sciences, Duke University School of Medicine, Durham, NC, USA; ${ }^{6}$ Department of Biostatistics and Bioinformatics, Duke University School of Medicine, Durham, NC, USA; ${ }^{7}$ Department of Health Policy and Management, University of North Carolina at Chapel Hill, Chapel Hill, NC, USA; ${ }^{8}$ Department of Psychiatry, Duke University School of Medicine, Durham, NC, USA; ${ }^{9}$ Duke University School of Nursing, Durham, NC, USA.
\end{abstract}

BACKGROUND: Little research has been done on primary care-based models to improve health care use after an emergency department (ED) visit.

OBJECTIVE: To examine the effectiveness of a primary care-based, nurse telephone support intervention for Veterans treated and released from the ED.

DESIGN: Randomized controlled trial with 1:1 assignment to telephone support intervention or usual care arms (ClinicalTrials.gov: NCTO 1717976).

SETTING: Department of Veterans Affairs Health Care System (VAHCS) in Durham, NC.

PARTICIPANTS: Five hundred thirteen Veterans who were at high risk for repeat ED visits.

INTERVENTION: The telephone support intervention consisted of two core calls in the week following an ED visit. Call content focused on improving the ED to primary care transition, enhancing chronic disease management, and educating Veterans and family members about VHA and community services.

MAIN MEASURES: The primary outcome was repeat ED use within 30 days.

KEY RESULTS: Observed rates of repeat ED use at 30 days in usual care and intervention groups were $23.1 \%$ and $24.9 \%$, respectively $(\mathrm{OR}=1.1 ; 95 \% \mathrm{CI}=0.7$, $1.7 ; P=0.6)$. The intervention group had a higher rate of having at least 1 primary care visit at 30 days $(\mathrm{OR}=1.6$, $95 \% \mathrm{CI}=1.1-2.3$ ). At 180 days, the intervention group had a higher rate of usage of a weight management program $(\mathrm{OR}=3.5,95 \% \mathrm{CI}=1.6-7.5)$, diabetes $/$ nutrition

Portions of this work were presented at the 2017 Academy Health Annual Research Meeting in New Orleans, LA.

Received November 28, 2018

Revised June 10, 2019

Accepted August 8, 2019

Published online September 5, 2019
$(\mathrm{OR}=1.8,95 \% \mathrm{CI}=1.0-3.0)$, and home telehealth services $(\mathrm{OR}=1.7,95 \% \mathrm{CI}=1.0-2.9)$ compared with usual care. CONCLUSIONS: A brief primary care-based nurse telephone support program after an ED visit did not reduce repeat $E D$ visits within 30 days, despite intervention participants' increased engagement with primary care and some chronic disease management services.

TRIALS REGISTRATION: ClinicalTrials.gov NCTO1717976.

KEY WORDS: randomized trials; Veterans; care management; health services research; primary care.

J Gen Intern Med 35(1):79-86

DOI: $10.1007 / \mathrm{s} 11606-019-05319-6$

() Society of General Internal Medicine (This is a U.S. government work and not under copyright protection in the U.S.; foreign copyright protection may apply) 2019

\section{INTRODUCTION}

The Veterans Health Administration (VHA) is the nation's largest integrated health care system and has more than two million emergency department (ED) visits annually. In the VHA, as in non-federal facilities, the majority of patients are discharged home from the ED rather than admitted to the hospital; however, approximately $20 \%$ of those discharged home re-visit the ED within 30 days. ${ }^{1,2}$ The risk of repeat visits is particularly high among patients with multiple chronic conditions and previous ED or hospital use. ${ }^{3}$ ED care is costly and health systems are motivated to improve care transitions after ED discharge to enhance quality and efficiency of primary care.

Optimizing care transitions is one central recommendation of the 2014 Geriatric Emergency Department Guidelines. ${ }^{4}$ Post-ED discharge telephone calls are one commonly used 
approach to support patients' transition(s) between the ED and post-ED settings. ${ }^{5}$ Content may focus on discharge-specific questions, including symptom management, medication reconciliation, and/or care coordination. ${ }^{6}$ To our knowledge, post-ED phone calls have not focused on educating patients on the patient-centered medical home (PCMH) model to manage urgent health concerns, including those related to chronic disease management and/or exacerbations. In VA, calls emphasizing care continuity in the PCMH are conducted by the patient's primary care team post-hospitalization. However, this approach has not been evaluated for post-ED discharge.

The primary objective of this study was to evaluate Discharge Information and Support for Patients receiving Outpatient care in the Emergency Department (DISPO ED), a primary care-based, nurse telephone support program for Veterans discharged home from the ED. To determine the program's impact on subsequent health care use, we compared repeat ED visit rates, hospitalizations, and engagement with outpatient clinical services for patients randomized to DISPO ED versus usual care. Our primary hypothesis was that participants in the nurse telephone support intervention would have a significantly lower rate of ED use in the 30 days following the index ED visit compared with usual care.

\section{METHODS}

The Institutional Review Board of the Durham VA Health Care System (DVAHCS) approved this study.

\section{Design and Setting}

This two-group randomized controlled trial was conducted at the DVAHCS. Methods were published previously. ${ }^{7}$ Participants were randomized 1:1 to nurse telephone support or usual care. Randomization was stratified by super user status $(\geq 3$ $\mathrm{ED}$ visits in the 6 months prior to the index ED visit). The primary outcome was repeat $\mathrm{ED}$ visits within 30 days.

\section{Participants and Recruitment}

Patients were eligible if they were discharged from the Durham VAHCS ED, received primary care at a Durham VAHCSaffiliated clinic, and were at high risk for repeat ED visits. We defined high risk as having $\geq 2$ chronic health conditions and at least one ED visit or hospitalization during the 6-month period before the index ED visit. Patients were excluded if they resided in an institutional care facility, were unable to communicate on the telephone, or lacked decision-making capacity with no legally authorized representative available to provide consent. Because monitoring patients at high risk of suicidality was beyond the scope of the study nurse, patients flagged as high suicide risk in their electronic medical record (EMR) were excluded. Eligible Veterans not returning to the ED within $24 \mathrm{~h}$ of the index ED visit were contacted by telephone the next business day and offered participation.
After providing verbal informed consent, participants or proxies completed a baseline assessment and were randomized to DISPO ED or usual care. Participants randomized to the intervention group had the option to identify a companion to participate with them.

\section{DISPO ED Intervention}

The intervention provided structured telephone support focused on three key areas: (1) improving the transition from ED to primary care; (2) enhancing chronic disease management; and (3) educating Veterans and family members about VHA's primary medical home model and other VA and community services. Each participant randomized to the intervention group received 2 core calls. Call 1 focused on identifying and addressing unmet needs related to the ED visit (e.g., medications, return precautions, follow-up needs). Call 2 focused on screening and referral for chronic disease management or preventive care programs (e.g., weight management) and also direct counseling for targeted needs (e.g., monitoring blood sugar). An optional 3rd call was scheduled during week 2 at the nurse and/or participant's request to answer additional questions, follow-up on referrals, etc. The nurse remained available to answer questions by phone for 30 days. Patient-specific recommendations were provided to primary care teams via progress notes in the EMR. At the end of the study, research team members reviewed patients' medical records to determine whether study recommendations had been completed as indicated by program enrollment or appointment attendance.

Fidelity in the delivery of the intervention was assessed via a second nurse listening in real time to intervention delivery calls and completing a checklist of intervention elements on a subset of calls periodically. The study nurse documented time spent on each call and preparation and post-processing time on a randomly selected subset $(\sim 20 \%)$.

\section{Usual Care}

All study participants received standard recommendations for follow-up care at the discretion of the individual ED provider, which often included a recommendation for patients to arrange a follow-up visit with their primary care team.

\section{Outcomes}

The primary outcome was any repeat ED use within 30 days following the index visit. This included all ED visits, whether or not the patient was admitted to the hospital. We asked participants about non-VHA health care use at 30- and 180day follow-up phone calls and included reported ED visits and hospitalizations that occurred outside of VHA if found in records for VA-financed care or confirmed by review of medical records at the treating facility. We also report ED visits that did not result in admission (treat and release ED visits), and ED visits and hospitalizations that occurred within 180 days of the index ED visit. 
We examined engagement with primary care, mental health, and chronic disease/preventive care and other specialty services using VHA administrative data files. MOVE! is a VHAbased weight management and health promotion program. TeleMOVE! is delivered via telephone and includes home monitoring telehealth technology.

Total VHA costs were calculated by aggregating utilization costs across VA and VA-financed care over the 180 days after the index ED visit for each subject. Total costs were adjusted to 2016 dollars. $^{8}$

Telephone surveys were conducted at baseline, 30 days, and 180 days to assess participant characteristics and non-VHA health care use. Demographic items were assessed at baseline, along with distance to nearest VA hospital or clinic, receipt of health care outside VA, health literacy ${ }^{21}$, and activities of daily living. The Healthy Days measure (HRQOL) was used to assess participant's perceived sense of well-being through four prompts: (1) assessment of self-rated health, (2) recall of physical days when health was not good, (3) recall of recent days when mental health was not good, (4) recall of recent activity limited days due to poor physical or mental health. ${ }^{9}$ Recent was defined as in the last 30 days. Outcome assessors were blinded to randomization.

\section{Sample Size}

The estimated sample size of 514 Veterans was based on detecting an 11-percentage point difference between arms in any ED use at 30 days (primary outcome) with $80 \%$ power and a two-sided $P$ value at the $P<0.05$ level. We used a difference in proportions analysis for the sample size calculation where we assumed a base ED visit rate of 0.32 (Proc Power, SAS Institute, Cary, NC). This sample size was inflated by $2 \%$ to account for anticipated deaths in the 30 days following the index ED based on data from a previous study. ${ }^{10}$

\section{Data Analysis}

Our primary hypothesis was that participants in the nurse telephone support intervention would have a significantly lower rate of ED use in the subsequent 30 days following the index ED visit compared with usual care. For participants with any repeat ED use, two authors (both clinicians) independently coded whether the primary reason for the first return VA ED visit was the same diagnosis or problem as the index ED visit, not the same but related, or an unrelated diagnosis or problem. The authors met to discuss and resolve discrepancies. One participant who was deceased at 30 days was excluded from utilization and cost analyses; all other randomly assigned participants had data available and were included. Statistical analyses were performed using SAS for Windows (version 9.4: SAS Institute, Cary, NC).

For dichotomous outcomes, multivariable logistic regression models were fit using SAS PROC LOGISTIC adjusting for the stratification variable. Model specification for the outcome of VA health care costs at 180 days was tested following recommendations by Manning and Mullahy. ${ }^{11}$ The optimal specification based upon fit tests for estimating differences in costs by arm, adjusting for the stratification variable, was a generalized linear model with a gamma distribution and log link. A 95\% confidence interval for the difference in mean costs was generated using 1000 bootstrapped samples.

\section{RESULTS}

\section{Baseline Participant Characteristics}

We identified 5951 potentially eligible ED patient-visits during our study enrollment period March 2014-January 2016 (Fig. 1). Daily lists of eligible patients were randomly sorted and screened until daily or weekly enrollment limits were reached. Enrollment limits were imposed to manage interventionist and other study staff workload. Of the 309 deemed ineligible at chart screen, the most common reason for exclusion was ineligible ED visit (not in study window, patient left before visit complete, psychiatric emergency care visit, admitted to the hospital; $n=173$ ). Of 1380 ED patient-visits screened by telephone, 1286 were eligible and 514 participants were enrolled and randomly assigned. One participant was determined to be ineligible after randomization and excluded; thus, there were 257 and 256 participants in the DISPO ED arm and usual care, respectively. Participant characteristics are shown in Table 1. Overall $78 \%$ of participants were male, half were of Black race, and one-quarter were ED super users. One hundred forty-nine $(29 \%)$ reported attempting to contact a provider prior to their index ED visit; of these, $83 \%$ were referred to the ED directly from a clinic $(n=44)$ or after a phone call with their primary care clinic $(n=$ 58 ) or nurse triage line $(n=22)$.

\section{Outcomes}

Observed rates of repeat ED use at 30 days in usual care and intervention groups were $23.1 \%$ and $24.9 \%$, respectively. Compared with usual care, there was no difference in repeat ED visits among intervention participants within 30 days $(\mathrm{OR}=1.1 ; 95 \% \mathrm{CI}=0.7,1.7 ; P=0.6)$ or 180 days $(\mathrm{OR}=$ $0.98 ; 95 \% \mathrm{CI}=0.7-1.4 ; P=0.9$ ). For the 117 patients with any repeat ED use within 30 days, two clinical coders had perfect agreement for $89(76 \%)$ of decisions as to how the index and first return ED visits were related (weighted kappa = 0.73). After adjudicating the remaining $28,26.5 \%$ of repeat ED visits were determined to be for the same diagnosis or problem $(n=31), 19.7 \%$ were not the same but related $(n=$ $23)$, and $53.8 \%(n=63)$ were unrelated. The most common reason for the first VA ED return visit within 30 days was a respiratory condition (e.g., cough, bronchitis, sinus, pneumonia; $n=17)$. There were no differences between arms for hospitalizations within 180 days.

The intervention group had a higher rate of having at least 1 primary care visit at 30 days $(\mathrm{OR}=1.6,95 \%$ 


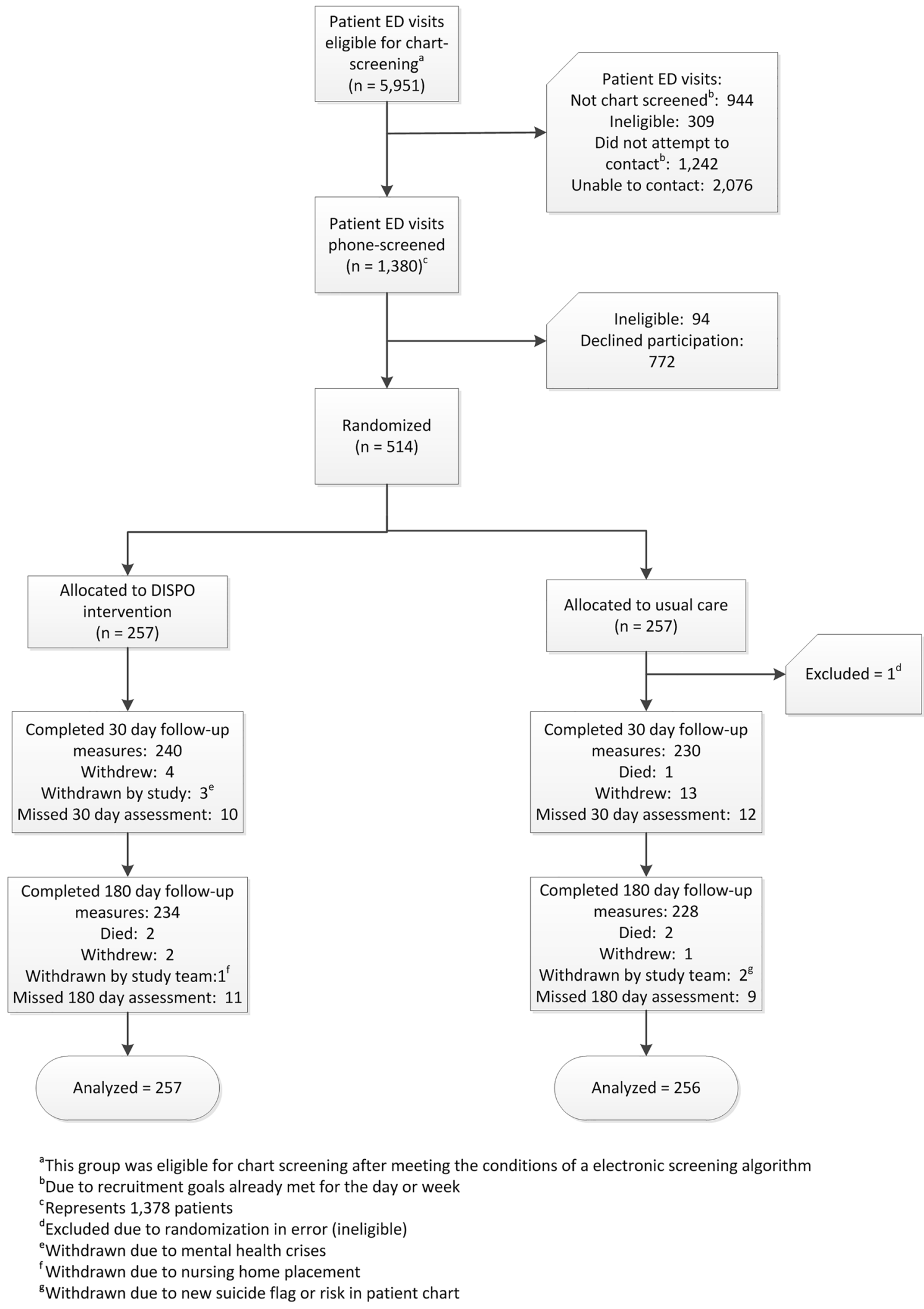

Figure 1 Consort.

$\mathrm{CI}=1.1-2.3)$. At 180 days, the intervention group had a higher rate of usage of weight management services $(\mathrm{OR}=3.5,95 \% \mathrm{CI}=1.6-7.5)$, diabetes/nutrition $(\mathrm{OR}=$ $1.8,95 \% \mathrm{CI}=1.0-3.0$ ), and home telehealth chronic disease management services $(\mathrm{OR}=1.7,95 \% \mathrm{CI}=1.0$ 2.9) compared with usual care. There were no differences between groups in total VA health care costs at 180 days (Table 2 ). 
Table 1 Baseline Characteristics

\begin{tabular}{|c|c|c|c|}
\hline & Total sample & DISPO ED intervention & Usual care \\
\hline & $N=\mathbf{5 1 3}$ & $N=\mathbf{2 5 7}$ & $N=256$ \\
\hline Age in years, mean $(\mathrm{SD})$ & $59.1(12.1)$ & $58.7(12.7)$ & $59.4(11.5)$ \\
\hline Male, $n(\%)$ & $399(77.8)$ & $197(76.7)$ & $202(78.9)$ \\
\hline Black or African American race, $n(\%)^{* \dagger}$ & $249(49.6)$ & $125(49.6)$ & $124(49.6)$ \\
\hline Hispanic/Latino ethnicity, $n(\%)^{*}$ & $15(3.1)$ & $9(3.7)$ & $6(2.5)$ \\
\hline Married or living together, $n(\%) *$ & $259(50.6)$ & $120(46.9)$ & $139(54.3)$ \\
\hline Education beyond high school, $n(\%)$ & $371(72.3)$ & $186(72.4)$ & $185(72.3)$ \\
\hline Inadequate income, $n(\%)^{*}$ & $228(46.2)$ & $121(48.6)$ & $107(43.9)$ \\
\hline Private insurance, $n(\%)^{*}$ & $110(21.5)$ & $53(20.7)$ & $57(22.4)$ \\
\hline \multicolumn{4}{|l|}{ Distance to nearest VA hospital or clinic, $n(\%)^{*}$} \\
\hline 0-20 miles & $208(41.1)$ & $111(43.9)$ & $97(38.3)$ \\
\hline $21-40$ miles & $163(32.2)$ & $68(26.9)$ & $95(37.5)$ \\
\hline 41 miles or more & $135(26.7)$ & $74(29.2)$ & $61(24.1)$ \\
\hline Receive health care outside VA, $n(\%)$ & $109(21.2)$ & $54(21.0)$ & $55(21.5)$ \\
\hline Inadequate health literacy, $n(\%) * *$ & $183(36.9)$ & $92(37.2)$ & $91(36.5)$ \\
\hline Chronic conditions, mean (SD) ${ }^{8}$ & $3.4(1.3)$ & $3.3(1.3)$ & $3.4(1.3)$ \\
\hline Fair or poor self-rated health, $n(\%)^{*}$ & $297(58.6)$ & $154(60.4)$ & $143(56.7)$ \\
\hline Frequent mental distress, $n(\%)^{*, 1}$ & $198(40.2)$ & $91(37.1)$ & $107(43.1)$ \\
\hline \multicolumn{4}{|l|}{ Number of ADL deficits, $n(\%)^{*}$, II } \\
\hline 0 & $360(70.7)$ & $175(68.4)$ & $185(73.1)$ \\
\hline $1-2$ & $106(20.8)$ & $57(22.3)$ & $49(19.4)$ \\
\hline $3-5$ & $43(8.4)$ & $24(9.4)$ & $19(7.5)$ \\
\hline "Super user", $n(\%)$ & $136(26.5)$ & $68(26.5)$ & $68(26.6)$ \\
\hline Contacted VA hospital before coming to ED, $n(\%)$ & $149(29.0)$ & $75(29.2)$ & $74(28.9)$ \\
\hline
\end{tabular}

SD, standard deviation: ADL, activities of daily living; ED, emergency department. Percentages adding to more than $100 \%$ are due to rounding

*Missing data (n): race (11), Hispanic/Latino ethnicity (34), married (1), inadequate income (20), private insurance (2), distance to VA (7), confidence in filling out medical forms (17), self-rated health (6), frequent mental distress (20), ADL deficits (4). Percentages are calculated excluding those with missing data from the denominator

FIncludes those who selected another race in addition to Black or African American

Response of "not at all," "a little bit," or "somewhat" to the question "How confident are you filling out medical forms by yourself?"

${ }^{\xi}$ Eligibility criteria - patients required to have 2 or more of 14 chronic conditions for study entry. Chronic conditions include anemia, asthma, congestive heart failure, chronic obstructive pulmonary disorder, chronic renal failure, dementia, depression, diabetes, hypertension, ischemic heart disease, peripheral vascular disease, obesity, osteoporosis, and post-traumatic stress disorder

'Between 14 and 30 days/month, self-reported mental health was "not good"

${ }^{\circledR}$ Needs some help or unable to do the following: bathe oneself, dress oneself, feed oneself, get from a bed to a chair by oneself, toilet by oneself

\section{Intervention Delivery}

Adherence to DISPO ED was exceedingly high: $99.2 \%$ of intervention participants completed call 1 and $98.1 \%$ completed both core calls. Half of participants received the optional third call, and $39 \%$ initiated at least one additional call with the study nurse during the 30-day intervention period. Overall median number of calls was 4.0/participant and average total intervention time was $39.5 \mathrm{~min} /$ participant (Fig. 2). For call 1, prep time averaged $11.5 \mathrm{~min}$ and post time averaged $11.1 \mathrm{~min}$. Eleven participants had companions (10 were a spouse or significant other) who were also involved in calls with the study nurse.

The study nurse made a total of 1200 recommendations for the 257 intervention participants (Table 3 ). The most common types were related to chronic disease management $(41.7 \%)$ and care facilitation (30.4\%). Participants accepted $33 \%$ of the chronic disease management recommendations, $54.5 \%$ of which were ultimately completed. When recommendations were not accepted, participants often cited the chronic disease or health behavior as an incidental health concern at the time or that they had tried the program before and had difficulty with access.

\section{DISCUSSION}

In this study of a brief primary care-based nurse telephone support program after an ED visit, we observed no differences in repeat ED visits within 30 days (primary outcome) or ED visits or hospitalizations within 180 days. The trial was adequately powered, the intervention was delivered with high fidelity, and utilization data were available on all participants; thus, we have confidence in our conclusion that the intervention did not reduce ED use in this population of VA users with multiple chronic conditions and previous ED or hospital use. Our negative results may be attributed to a variety of factors. Development of the DISPO ED intervention was guided by a conceptual model which emphasized the importance of identifying and addressing unmet health care needs and chronic disease management in affecting ED use. ${ }^{12-14}$ A more recent conceptual model, published after study enrollment was completed, emphasizes the role of socio-environmental and individual factors such as personal preferences, and previous health care experiences as primary drivers of decisionmaking around episodes of acute, unscheduled care. ${ }^{15}$ Indeed, we observed high rates of individual-level social stressors in this population, such as $46 \%$ reporting inadequate income. A 
Table 2 ED Visits, Hospitalizations, and Engagement with VA Clinical Service

\begin{tabular}{|c|c|c|c|c|}
\hline & $\begin{array}{l}\text { DISPO ED intervention, } \\
n=257^{*}\end{array}$ & $\begin{array}{l}\text { Usual care, } \\
n=255\end{array}$ & $\overline{\mathrm{OR}}(\mathbf{9 5 \%} \mathrm{CI})^{\dagger}$ & $P$ value \\
\hline \multicolumn{5}{|l|}{ ED visits within 30 days } \\
\hline Repeat ED use, $n$ (\% yes) & $64(24.9)$ & $59(23.1)$ & $1.1(0.7,1.7)$ & 0.63 \\
\hline Treat and release ED visits, $n$ (\% yes) & $57(22.2)$ & $50(19.6)$ & $1.2(0.8,1.8)$ & 0.46 \\
\hline \multicolumn{5}{|l|}{ ED visits within 180 days } \\
\hline Repeat ED use, $n$ (\% yes) & $160(62.3)$ & $160(62.7)$ & $0.98(0.7,1.4)$ & 0.91 \\
\hline Treat and release ED visit, $n$ (\% yes) & $145(56.4)$ & $146(57.3)$ & $0.97(0.7,1.4)$ & 0.85 \\
\hline \multicolumn{5}{|l|}{ Hospitalizations ${ }^{\star}$ within 180 days } \\
\hline Hospitalization, n (\% yes) & $68(26.5)$ & $61(23.9)$ & $1.1(0.8,1.7)$ & 0.50 \\
\hline \multicolumn{5}{|c|}{ Engagement with VA Clinical Services within 30 days } \\
\hline Primary care visit, $n(\%)$ & $128(49.8)$ & $98(38.4)$ & $1.6(1.1,2.3)$ & 0.010 \\
\hline Mental health visit, $n(\%)$ & $42(16.3)$ & $45(17.6)$ & $0.9(0.6,1.4)$ & 0.70 \\
\hline \multicolumn{5}{|c|}{ Engagement with VA Clinical Services within 180 days } \\
\hline Primary care visit, $n(\%)$ & $237(92.2)$ & $223(87.5)$ & $1.7(0.9,3.1)$ & 0.077 \\
\hline Mental health, $n(\%)$ & $106(41.2)$ & $101(39.6)$ & $1.1(0.8,1.5)$ & 0.70 \\
\hline MOVE or TeleMOVE! visit, $n(\%)$ & $29(11.3)$ & $9(3.5)$ & $3.5(1.6,7.5)$ & 0.002 \\
\hline Diabetes or nutrition visit, $n(\%)$ & $40(15.6)$ & $24(9.4)$ & $1.8(1.0,3.0)$ & 0.037 \\
\hline Care Coordination and Home Telehealth (CCHT) & $42(16.3)$ & $26(10.2)$ & $1.7(1.0,2.9)$ & 0.042 \\
\hline Medication management visit, $n(\%)$ & $72(28.0)$ & $68(26.7)$ & $1.1(0.7,1.6)$ & 0.73 \\
\hline Sleep visit, $n(\%)$ & $41(16.0)$ & $33(12.9)$ & $1.3(0.8,2.1)$ & 0.33 \\
\hline Rehabilitation services visit, $n(\%)$ & $118(45.9)$ & $107(42.0)$ & $1.2(0.8,1.7)$ & 0.37 \\
\hline Orthopedics visit, $n(\%)$ & $69(26.8)$ & $58(22.7)$ & $1.2(0.8,1.9)$ & $\begin{array}{l}0.28 \\
P \text { value }\end{array}$ \\
\hline $\begin{array}{l}\text { VA and VA-financed care costs within } 180 \text { days } \\
\text { Total health care costs } \S, \text { mean (SD) }\end{array}$ & $38,273(57,836)$ & $31,696(46,295)$ & $5993(-2995,15,158)$ & 0.068 \\
\hline
\end{tabular}

n, number; SD, standard deviation; CI, confidence interval; ED, emergency department

* Observed rates and/or means (SD). One participant out of the 256 randomized to usual care was excluded in utilization outcomes due to death within 30 days of index emergency department visit

† Model results adjusted for baseline emergency department "super user" status. Odds ratios (OR) from logistic regression models reported for dichotomous outcomes. Reference group = usual care arm

Including observation days

${ }^{\S}$ Costs in 2016 US dollars. Observed median costs: DISPO ED intervention \$17,599; usual care \$15,293

'Estimated using generalized linear model with gamma distribution and log link, adjusted for baseline emergency department "super user" status. Reference group = usual care arm. 95\% confidence intervals calculated using 1000 bootstrapped samples

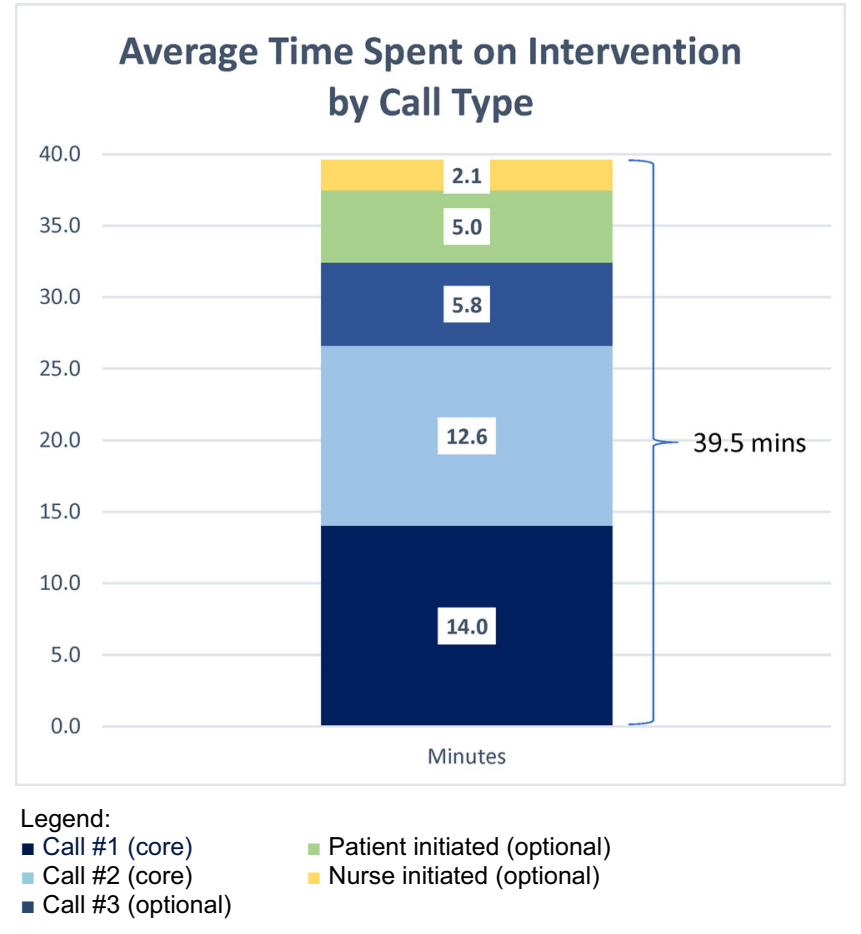

Figure 2 Average time (minutes) spent on intervention. a call no. 1 (core), patient initiated (optional), call no. 2 (core), nurse initiated (optional), $\square$ call no. 3 (optional). greater focus on addressing psychological health may have been warranted, as $40 \%$ of participants reported frequent mental distress in the month prior to enrollment. Finally, identifying unmet needs requires having a way to address them within the context of the health system. We found that referrals to the ED from outpatient providers were common; therefore, a paired system-level intervention to enhance options for and access to acute care in other settings besides the ED may have been beneficial.

Intervention participants were more likely to have a primary care visit and to receive some preventive and/or chronic disease management services, compared with usual care. This is consistent with the actions of the study nurse, which were often focused on facilitating these types of care. Despite this increased use of primary care and disease management services, we did not observe any reductions in hospitalizations or costs within 180 days. Our goal was to address underlying contributing factors of some ED visits, for example, poorly controlled diabetes leading to predisposition to urinary tract infections and dehydration. However, it is possible that the chronic disease management programs referred to by the intervention nurse did not address the same issues leading to ED use. Additionally, our review of reasons for return ED visits found that more than half were for issues that were 
Table 3 Summary of Study Nurse Actions and Recommendations ( $n=257$, DISPO ED Group Only)

\begin{tabular}{|c|c|c|c|}
\hline Actions and recommendations & $\begin{array}{l}\text { Issued by study nurse } \\
n(\%)\end{array}$ & $\begin{array}{l}\text { Accepted by participant } \\
n(\%)\end{array}$ & $\begin{array}{l}\text { Completed* } \\
n(\%)\end{array}$ \\
\hline Chronic disease management and care coordination $^{\dagger}$ & $500(41.7)$ & $165(33.0)$ & $90(54.5)$ \\
\hline $\begin{array}{l}\text { Facilitation of care from existing provider and direct ordering } \\
\text { of tests, medications or supplies }\end{array}$ & $365(30.4)$ & $361(98.9)$ & $318(88.1)$ \\
\hline Provision of printed educational materials & $110(9.2)$ & $110(100)$ & $110(100)$ \\
\hline VA benefits assistance & $83(6.9)$ & $83(100)$ & $80(96.4)$ \\
\hline Referral for consultation with new clinic or provider & $71(5.9)$ & $67(94.4)$ & $53(79.1)$ \\
\hline Referral to non-VA medical services or community programs & $41(3.4)$ & $38(92.7)$ & $34(89.5)$ \\
\hline Mental health care facilitation & $30(2.5)$ & $23(76.7)$ & $21(91.3)$ \\
\hline Total & $1200(100)$ & $847(70.6)$ & $706(83.4)$ \\
\hline
\end{tabular}

* "Completed" refers to actions or recommendations that were completed by the study nurse alone, initiated by the study nurse and accepted by the responsible party, or a program enrollment or appointment completed date was found in the participant medical record

HInterpretation: 500 out of 1200 study nurse actions or recommendations were in the category of chronic disease management and care coordination; 165 out of the 500 (33.0\%) of these were initially accepted by the participant, and 90 out of the 165 (54.5\%) were ultimately completed

unrelated to the initial visit, underscoring the heterogeneity of needs in this population. Also, it may require a longer time frame to realize cost-savings from positive behavior change and improved disease control. ${ }^{16}$

A novel aspect of our study design was to allow dyadic enrollment of both patients and companions; however, there was low uptake of this feature. Although it is widely acknowledged that medically complex patients can benefit from caregiver or family involvement in care transitions, ${ }^{12-14,} 17$ our experience highlights that some individuals may lack strong social networks and others may not find it comfortable or necessary to involve family members in care coordination interactions. Further studies should explore whether enhanced non-medical support from family members or communitybased providers can have a positive impact on primary carebased telephone support interventions.

Acknowledging the time constraints on nurses in primary care, our intervention was designed to be brief and we accomplished that goal with mean total call time of less than $40 \mathrm{~min}$ per participant. A more intensive intervention, in terms of number of contacts and/or duration, may have been more effective. A recent systematic review of ED interventions focused on older adults found evidence that studies using multi-strategy approaches and those with a more comprehensive structure were more likely to be associated with improved outcomes. ${ }^{18}$ It should be noted that highly time-intensive interventions can present challenges for widespread adoption because of the numbers of individuals potentially affected. For example, approximately 1 in 4 of all ED patients met our current study's definition of being high risk and, thus, was eligible to receive the DISPO ED intervention. ${ }^{19} \mathrm{~A}$ highintensity intervention would likely need to be targeted to a smaller proportion of ED patients in order to be cost-effective.

Several limitations warrant acknowledgement. The study was conducted in a single VAHCS and may not be applicable to patients in other health care systems, particularly ones in which not all patients have access to primary care medical homes. We focused on patients who were at high risk for a repeat $\mathrm{ED}$ visit; however, these patients may or may not be the same as those who would be most likely to benefit from this type of intervention. Consistent with our pragmatic design, we measured outcomes that were relevant to a broad population, rather than focused on a single disease or complaint; however, these may not have been optimally responsive measures in this context. A recent report by the National Quality Forum highlighted the need for development and testing of new measures to assess patient experience following an ED visit. ${ }^{20}$

In conclusion, a brief nurse-led telephone support program after an ED visit did not reduce repeat ED visits, despite intervention participants' increased engagement with primary care and some chronic disease management services. In clinical practice, interventions to successfully reduce return ED visits will require more than brief telephone follow-up calls and will need to focus on psychological and social factors as much as unmet health care needs.

Acknowledgments: We would like to thank Lesa Powell and Teresa Hinton for their tireless efforts on behalf of the DISPO ED study, and our clinical advisory board members (Eleanor McConnell, William Knaack, Wendy Henderson, and Jorge Cortina) for their significant intellectual contributions to the research.

Corresponding Author: Susan N. Hastings, MD, MHSc; Center of Innovation to Accelerate Discovery and Practice Transformation Durham VA Health Care System, HSR\&D, Fulton Street, Durham, NC, USA (e-mail: susan.hastings@va.gov).

Funding Information This work was supported by the United States (U.S.) Department of Veterans Affairs, Health Services Research and Development Service (IIR 12-052; HX000976A) and by the Center of Innovation to Accelerate Discovery and Practice Change (CIN 13-410) at the Durham VA Health Care System. KES also received support from the National Institute on Aging, Duke Claude D. Pepper Older Americans Independence Center, NIA P3OAG028716.

\section{Compliance with Ethical Standards:}

The Institutional Review Board of the Durham VA Health Care System (DVAHCS) approved this study.

Conflict of Interest: The authors declare that they do not have a conflict of interest. 
Disclaimer: The contents do not represent the views of the U.S. Department of Veterans Affairs or the United States Government.

\section{REFERENCES}

1. Hastings SN, Smith VA, Weinberger M, Schmader KE, Olsen MK Oddone EZ. Emergency department visits in Veterans Affairs medical facilities. Am J Manag Care. 2011;17(6 Spec No.):e215-223.

2. Dharmarajan $\mathbf{K}$, Gin $\mathbf{L}$, Bierlein $\mathbf{M}$, et al. Outcomes after observation stays among older adult Medicare beneficiaries in the USA: retrospective cohort study. BMJ. 2017;357:j2616.

3. Hastings SN, Oddone EZ, Fillenbaum G, Sloane RJ, Schmader KE. Frequency and predictors of adverse health outcomes in older Medicare beneficiaries discharged from the emergency department. Medical Care. 2008;46(8):771-777.

4. American College of Emergency Physicians, American Geriatrics Society, Emergency Nurses Association, Society for Academic Emergency Medicine, Geriatric Emergency Department Guidelines Task Force. Geriatric emergency department guidelines. Ann Emerg Med. 2014;63(5):e7-25.

5. Atzema CL, Maclagan LC. The transition of care Between emergency department and primary care: A scoping study. Acad Emerg Med. 2017;24(2):201-215.

6. Morse L, Xiong L, Ramirez-Zohfeld V, Dresden S, Lindquist LA. Telefollow-up of older adult patients from the Geriatric Emergency Department Innovation (GEDI) Program. Geriatrics (Basel). 2019;4(1).

7. Hastings SN, Betts E, Schmader KE, et al. Discharge information and support for veterans receiving outpatient care in the emergency department: study design and methods. Contemp Clin Trials. 2014;39(2):342350.

8. Dunn A, Grosse SD, Zuvekas SH. Adjusting Health Expenditures for Inflation: A Review of Measures for Health Services Research in the United States. Health Serv Res. 2018;53(1):175-196.

9. Centers for Disease Control and Prevention. Measuring Healthy Days. 2000. Available at: http://www.cdc.gov/hrqol/pdfs/mhd.pdf. Accessed July 11, 2019.

10. Hastings SN, Schmader KE, Sloane RJ, Weinberger M, Goldberg KC Oddone EZ. Adverse health outcomes after discharge from the emergency department-incidence and risk factors in a veteran population. J Gen Intern Med. 2007;22(11):1527-1531.

11. Manning WG, Mullahy J. Estimating log models: to transform or not to transform? J Health Econ. 2001;20(4):461-494.

12. Caplan GA, Williams AJ, Daly B, Abraham K. A randomized, controlled trial of comprehensive geriatric assessment and multidisciplinary intervention after discharge of elderly from the emergency department-the DEED II study. J Am Geriatr Soc. 2004;52(9):1417-1423.

13. Gagnon AJ, Schein C, McVey L, Bergman H. Randomized controlled trial of nurse case management of frail older people. J Am Geriatr Soc. 1999;47(9): 1118-1124.

14. Guttman A, Afilalo M, Guttman R, et al. An emergency departmentbased nurse discharge coordinator for elder patients: does it make a difference? Acad Emerg Med. 2004;11(12):1318-1327.

15. Pines JM, Lotrecchiano GR, Zocchi MS, et al. A Conceptual Model for Episodes of Acute, Unscheduled Care. Ann Emerg Med. 2016;68(4):484491 e483.

16. Lee JY, Muratov S, Tarride JE, Holbrook AM. Managing High-Cost Healthcare Users: The International Search for Effective EvidenceSupported Strategies. J Am Geriatr Soc 2018.

17. Naylor MD, Brooten D, Campbell R, et al. Comprehensive discharge planning and home follow-up of hospitalized elders: a randomized clinical trial. JAMA. 1999;281(7):613-620.

18. Hughes JM, Freiermuth CE, Shepherd-Banigan M, et al. Emergency Department Interventions for Older Adults: A Systematic Review. J Am Geriatr Soc. 2019.

19. Miller KEM, Duan-Porter W, Stechuchak KM, et al. Risk stratification for return emergency department visits among high-risk patients. Am J Manag Care. 2017;23(8):e275-e279.

20. National Quality Forum. Emergency department transitions of care: A quality measurement framework. 2017.

21. Chew LD, Bradley KA, Boyko EJ.. Brief questions to identify patients with inadequate health literacy. Fam Med. 2004;36(8):588-594.

Publisher's Note Springer Nature remains neutral with regard to jurisdictional claims in published maps and institutional affiliations. 